\title{
Purely Catalytic P Systems over Integers and Their Generative Power
}

Artiom Alhazov ${ }^{1}$, Omar Belingheri ${ }^{2}$, Rudolf Freund ${ }^{3}$, Sergiu Ivanov ${ }^{4}$, Antonio E. Porreca ${ }^{2}$, and Claudio Zandron ${ }^{2}$

1 Institute of Mathematics and Computer Science Academy of Sciences of Moldova

Str. Academiei 5, Chişinău, MD 2028, Moldova

E-mail: artiom@math.md

2 Dipartimento di Informatica, Sistemistica e Comunicazione

Università degli Studi di Milano-Bicocca

Viale Sarca 336/14, 20126 Milano, Italy

E-mail: $\{$ o.belingheri@campus, porreca@disco, zandron@disco $\}$.unimib.it

3 Faculty of Informatics, TU Wien

Favoritenstraße 9-11, 1040 Vienna, Austria

E-mail: rudi@emcc.at

4 Université Paris Est, France

E-mail: sergiu.ivanov@u-pec.fr

Summary. We further investigate the computing power of the recently introduced $\mathrm{P}$ systems with $\mathbb{Z}$-multisets (also known as hybrid sets) as generative devices. These systems apply catalytic rules in the maximally parallel way, even consuming absent non-catalysts, effectively generating vectors of arbitrary (not just non-negative) integers. The rules may be made inapplicable only by dissolution rules. However, this releases the catalysts into the immediately outer region, where new rules might become applicable to them. We discuss the generative power of this model. Finally, we consider the variant with mobile catalysts.

\section{Introduction}

Membrane systems (cell-like, with symbol-objects) have traditionally been viewed as collections of hierarchically arranged multiset processors [12]. In the list of open problems disseminated in 2015 [11], Gheorghe Păun suggested going beyond the traditional setting where symbol multiplicities in multisets are restricted to non-negative integers. One suggested approach [6] defines generalized multisets as taking multiplicities from arbitrary finitely generated, totally ordered commutative groups.

In work [3], a different approach is taken: only catalytic rules are allowed, and the applicability of a rule only depends on presence of the corresponding catalyst 
in the given region. Consuming an absent non-catalyst makes its multiplicity negative. While in [3] it was already established that such model is not universal, we found it interesting to investigate its generative power more precisely.

Since the number of catalysts remains finite and does not change throughout the computation, this induces a finite set of "rule teams" which can be applied in parallel in one step. The virtual absence of applicability conditions and the finiteness of the "teams" hints at the possibility of seeing them as integer vectors; in this case the P system itself can be seen as evolving by sequentially adding such vectors (possibly having negative components) to the contents of its membranes. Paper [2] compares this general model to vector addition systems [5, 9] (adapted to allow negative vector components [8]) and blind register machines [7].

Here we return to the particular model from [3], discussing the lower bound of its generative power and giving some results on the variant with target indications.

\section{Preliminaries}

The reader is assumed to be familiar with the basic notions of formal languages and membrane computing; see [13] for a comprehensive introduction to both. We only remark that, as common in membrane computing, multisets in $O^{\circ}=\mathbb{N}^{O}$ are represented by strings in $O^{*}$, keeping in mind that the order of symbols is not relevant.

\subsection{Extending Multisets}

To represent also negative multiplicities, multisets must be extended. A $\mathbb{Z}$-multiset, allowing integer multiplicities (called a hybrid set in [4]) would be from $\mathbb{Z}^{O}$; it can be represented by a string in $\left(O \cup O^{-}\right)^{*}$, where $O^{-}=\left\{a^{-} \mid a \in O\right\}$ is a set of symbols that represents objects in multiplicity "negative one". Note that, as opposed to $\mathrm{P}$ systems with matter-antimatter [1], symbol $a^{-}$here is not an actual object, but simply a convenient way to represent a deficit of $a$, and the actual multiplicity of $a$ represented by a string $w$ is $|w|_{a}-|w|_{a^{-}}$. We also do not distinguish between notations $a^{-k}$ and $\left(a^{-}\right)^{k}$. The superscript ${ }^{-}$can be used as a morphism, producing a multiset with opposite multiplicities, e.g., $\left(a^{k}\right)^{-}$represents the same $\mathbb{Z}$-multiset as the one in the previous sentence. As the strings here are only used to represent $[\mathbb{Z}-]$ multisets, we may write an equality sign between the strings representing the same $[\mathbb{Z}-]$ multiset. For conciseness, let us use the notation $O^{\bullet}=\left(O \cup O^{-}\right)^{*}$. Finally, since it will be always clear from the context, we may call an element of $O^{\bullet}$ "multiset", omitting the word "representing". Assuming an order is fixed on $O$, for $u \in O^{\bullet}$, vector $\left(|u|_{a}-|u|_{a^{-}}\right)_{a \in O}$ is denoted by $\psi_{O}(u)$; the subscript $O$ may be omitted when it is clear from the context. This vector is called the Parihkh image of $u$. 


\subsection{Linear Sets}

The linear set generated by a set of vectors $A=\left\{\mathbf{a}_{i} \mid 1 \leq i \leq d\right\} \subset \mathbb{Z}^{n}$ and an offset $\mathbf{a}_{0} \in \mathbb{Z}^{n}$ is defined as follows:

$$
\left\langle A, \mathbf{a}_{0}\right\rangle_{\mathbb{N}}=\left\{\mathbf{a}_{0}+\sum_{i=1}^{d} k_{i} \mathbf{a}_{i} \mid k_{i} \in \mathbb{N}, 1 \leq i \leq d\right\} .
$$

If the offset $\mathbf{a}_{0}$ is the zero vector, we will call the corresponding linear set homogeneous; we also will use a short notation $\langle A\rangle_{\mathbb{N}}=\langle A, \mathbf{0}\rangle_{\mathbb{N}}$.

We use the notation $\mathbb{Z}^{n} L I N_{\mathbb{N}}=\left\{\left\langle A, \mathbf{a}_{0}\right\rangle_{\mathbb{N}} \mid A \in\left(\mathbb{Z}^{n}\right)^{d}, \mathbf{a}_{0} \in \mathbb{Z}, m \in \mathbb{N}\right\}$, to refer to the class of all linear sets. Semilinear sets are defined as finite unions of linear sets. We use the notations $\mathbb{Z}^{n} S L I N_{\mathbb{N}}$ to refer to the classes of semilinear sets of $n$-dimensional vectors. In case no restriction is imposed on the dimension, $n$ is replaced by $*$. We may omit $n$ if $n=1$. A finite union of linear sets which only differ in the starting vectors is called uniform semilinear:

$$
\begin{aligned}
& \mathbb{Z}^{n} S L I N_{\mathbb{N}}^{U}=\left\{\bigcup_{\mathbf{b} \in B}\langle A, \mathbf{b}\rangle_{\mathbb{N}} \mid A \in\left(\mathbb{Z}^{n}\right)^{d}, B \in\left(\mathbb{Z}^{n}\right)^{k}, d, k \in \mathbb{N}\right\} \\
& =\left\{\left\{\mathbf{b}+\sum_{i=1}^{d} k_{i} \mathbf{a}_{i} \mid k_{i} \in \mathbb{N}, 1 \leq i \leq d\right\} \mid A \in\left(\mathbb{Z}^{n}\right)^{d}, B \in\left(\mathbb{Z}^{n}\right)^{k}, d, k \in \mathbb{N}\right\} .
\end{aligned}
$$

Let us denote these sets by $\langle A, B\rangle_{\mathbb{N}}$.

\section{Purely Catalytic P Systems over Integers}

In purely catalytic $\mathrm{P}$ systems over integers the set of objects is a disjoint union of catalysts $C$ and the regular objects $O$. The regular objects are allowed to have any integer multiplicity, while the catalysts are only allowed to appear in a nonnegative number of copies.

The rules can be of the two following types:

- catalytic rules: $c u \rightarrow c v$, where $c \in C$ and $u, v \in O^{*}$;

- catalytic rules with dissolution: $c u \rightarrow c v \delta$, where $c \in C, u, v \in O^{*}$, and $\delta \notin$ $C \cup O$ is the symbol indicating membrane dissolution.

The rules applied in parallel cannot involve more catalysts than available in the system; the multiplicities of regular objects, on the other hand, do not influence the applicability of rules. An application of a rule $c u \rightarrow c v$ in a region containing $c w\left(c \in C, u, v \in O^{*}, w \in O^{\bullet}\right.$ produces $c w(c u)^{-} c v=c w v\left(u^{-}\right)$, or, in terms of vectors, ignoring the catalyst, vector $\psi(w)+\psi(v)-\psi(u)$ is represented by the contents of that region after the rule has been applied. An application of a rule $c u \rightarrow c v \delta$ produces the same effect, and then dissolves the enclosing membrane, moving the contents of the dissolved membrane into the parent membrane.

Purely catalytic P systems over integers evolve under the maximally parallel semantics, so each catalyst enters exactly one rule (non-deterministically chosen), unless the given region has no rules associated with this catalyst. By 
$Z^{d} O_{\mathbb{Z}} P_{m}\left(p_{c a t}, \delta\right)$ we denote the family of sets of $d$-dimensional vectors of integers generated by purely catalytic $\mathrm{P}$ systems over integers with dissolution, at most $m$ membranes and at most $k$ catalysts. If any of parameters $d, m, k$ is unbounded, it is replaced by $*$ in the notation.

We also use notations for extended features (listed in parentheses in the notation of the sets of $\mathbb{Z}$-vectors generated by the corresponding families of $\mathrm{P}$ systems). Target indications, denoted by tar, allow the non-catalysts to be sent to a different membrane. In the right side of the rules, sending object $a$ is written by $(a, t a r)$, where $\operatorname{tar} \in\{$ out $\} \cup\left\{i n_{j} \mid 1 \leq j \leq m\right\} ; j$ here is a label of immediately inner membrane. In this paper, we may write $\operatorname{tar}_{n}$ in the notation of a set of $\mathbb{Z}$-vectors generated by a family of $\mathrm{P}$ systems; this generalization reflects the possibility to assign targets even to negative multiplicites of objects.

Another feature is mobile catalysts [10], i.e., targets may also be associated to the catalysts, and thus the catalysts move across the membrane structure; we denote this feature by mpcat $_{k}$ since the systems we consider are purely catalytic. We use the plus sign between the features of catalytic mobility and dissolution when it is allowed for the same rule to move a catalyst and to dissolve the membrane currently containing it.

\section{Results}

\subsection{Simplifications and Observations}

First, we would like to explicitly allow rules of the form $c \rightarrow c x,\left(c \in C, x \in O^{\bullet}\right)$, i.e., the multiset of regular objects in the left side being empty. This does not change the model, since any $\mathbb{Z}$-multiset $x$ can be written as $u\left(v^{-}\right), u, v \in O^{*}$, and, fixing some $a \in O, c \rightarrow c x$ is equivalent to $c a u \rightarrow a v$. Moreover, any rule $c u \rightarrow c v$ is equivalent to $c \rightarrow c u\left(v^{-}\right)$, so it suffices to only consider rules of types $c \rightarrow c x$ and $c \rightarrow c x \delta\left(c \in C, x \in O^{\bullet}\right)$.

Second, notice that it is enough to start with a single catalyst in any region, because it can perform the role of any number of catalysts, and if multiple catalysts are initially in the same region, they will always stay in the same region (possibly, merged with others). Indeed, take an arbitrary region of an arbitrary purely catalytic $\mathrm{P}$ system over integers, say, it has catalysts $c_{i}, 1 \leq i \leq d$, and each catalyst $c_{i}$ has associated rules $c_{i} \rightarrow c_{i} x_{i, j}, 1 \leq j \leq n_{i}$, where $x_{i, j} \in O^{\bullet} \cup O^{\bullet} \delta$. Note that if none of the catalysts has associated rules, then they are equivalent to a single catalyst with no associated rules, so in the following we assume the contrary. If some catalyst $c_{i}$ has no associated rules, it is then equivalent to it having associated a single rule $c_{i} \rightarrow c_{i}$, i.e., $x_{i, 1}=\lambda$ and $n_{i}=1$, so in the following we assume $n_{i} \geq 1$ for $1 \leq i \leq d$. We can now replace all these catalysts by a single catalyst $c$ having associated the following set of rules:

$$
\left\{c \rightarrow c x_{1, j_{1}} \cdots x_{d, j_{d}} \mid 1 \leq j_{i} \leq n_{i}, 1 \leq i \leq d\right\} .
$$


On the other side, no catalyst in some region is equivalent to one catalyst with no associated rules. Therefore, without restricting the generality, in the following we assume that in the initial configuration of an arbitrary purely catalytic P system over integers, each membrane region $i, 1 \leq i \leq m$, contains precisely one catalyst, and we can call it $c_{i}$.

Third, notice that no information enters membranes, so the outer regions cannot affect the inner regions in any way. Hence, if the output region $i_{0}$ is not the skin, then only the membrane substructure inside $i_{0}$, including $i_{0}$ is relevant for the result, and other membranes are irrelevant and may be removed without affecting the result, making $i_{0}$ the skin (unless some rule in some removed membrane had applicable rules, but could never be dissolved, in which case the generated set of vectors is empty, which is a degenerate case). So in the following, we assume that the output region is always the skin.

Fourth, every elementary membrane having no rules associated to the catalysts available there may be removed from the system without affecting the result (unless it is the output membrane, in which case a singleton is generated, which is a degenerate case), so in the following we assume that each elementary membrane has some applicable rules. Clearly, the P system will not reach the halting until this membrane is dissolved.

Consider this reasoning starting from the elementary membranes outside, by induction. Take any non-elementary membrane $i$ which becomes elementary during a computation. Assume $i$ is not dissolved (i.e., it has no rules associated to any of the catalysts that were placed within the membrane substructure inside $i$, including $i$ ), but it is not the output membrane. Then all the computation in the membrane substructure inside $i$, including $i$, does not contribute to the result, and can be removed from the system without affecting the result.

As a summary of the fourth observation, without restricting the generality (except, possibly the degenerate cases generating the empty set or some singleton), we may assume that any purely catalytic $\mathrm{P}$ system over integers has applicable rules associated to all elementary membranes, and all membranes except the skin must be dissolved at some moment during the computation.

Finally, for every region except the skin, a catalyst $c_{i}$ without associated rules is equivalent to a catalyst with a rule $c_{i} \rightarrow c_{i}$. Hence, without restricting the generality, we may assume that the catalysts are never idle before the halting is reached. Clearly, (excluding the degenerate case generating the empty set), the skin should have no rules associated to any catalyst of the system.

We would like to note that even without pruning the membrane structure by removing membrane substructures not contributing to the result, the membrane structure obtained at halting (if at all reachable) is unique.

We recall that in [2], the following generalization approach is taken: There is a finite number of reachable membrane structures. These could be used as states of a sequential P system, which may be obtained, separately for each membrane structure, by combining the behavior of all catalysts in all regions of the $\mathrm{P}$ system. Indeed, having fixed a reachable membrane structure, we know which membranes 
have been dissolved, and thus the resulting location of each catalyst. Then, for each catalyst, associated rules in its current location are considered and combined, similarly to the second observation above, but globally. Having obtained a sequential system, the catalyst is no longer needed. Then, in [2] it was shown that such a generalization is nothing else but a sequential blind vector addition system with states, and it was claimed that it characterizes precisely the family of all semilinear vectors of integers.

Indeed, in this way any purely catalytic P system over integers can be substituted by a sequential blind vector addition system with states, so the upper bound of the family of all semilinear sets of vectors of integers, or, equivalently, the family of all integer vector sets, generated by blind register machines, holds. However, the reverse is not necessarily true, i.e., it does not follow that for any sequential blind vector addition system with states there would exist an equivalent purely catalytic $\mathrm{P}$ system over integers.

Another result in [2] has been obtained for integer vector addition $\mathrm{P}$ systems, namely Theorem 5 . That model has been shown to characterize exactly the uniform semilinear sets. However, since in the model of integer vector addition P systems, as opposed to purely catalytic P systems over integers, there is no concept of a catalyst, dissolving a membrane only disables rules of that region, without enabling rules that, in purely catalytic $\mathrm{P}$ systems over integers, are contained in the parent region and associated to the catalysts that were in the dissolved region. Hence, the characterization from Theorem 5 of [2] has no direct implication on the power of purely catalytic $\mathrm{P}$ systems over integers.

Therefore, at this point in the present paper we would like to definitely deviate into the particularities of how dissolution affects the computation, and the lower bounds.

\subsection{Generative Power}

We recall that we discuss the family of integer vector sets generated by purely catalytic P systems over integers, with the usual halting condition.

Since the output region cannot be dissolved by definition and any other applicable rule can never be stopped, single-membrane purely catalytic $\mathrm{P}$ systems over integers are degenerate:

$$
Z^{d} O_{\mathbb{Z}} P_{1}\left(\text { pcat }_{*}, \delta\right)=\{\emptyset\} \cup\left\{\{v\} \mid v \in \mathbb{Z}^{d}\right\} .
$$

For simplicity, we will not mention these degenerate cases while considering multiple membranes.

With two membranes, a characterization is still straightforward:

$$
Z^{d} O_{\mathbb{Z}} P_{2}\left(\text { pcat }_{*}, \delta\right)=\mathbb{Z}^{d} S L I N_{\mathbb{N}}^{U} .
$$

Indeed, let $A$ be the finite set of vectors corresponding to the non-dissolving rules in the elementary membranes, and let $B$ be the finite set of sums of two vectors: 
the one corresponding to the initial configuration and vectors corresponding to the dissolving rules in the elementary membrane; the skin should have no rules. If the catalyst in the elementary membrane is $c_{2}$, then the correspondence mentioned above is $c_{2} \rightarrow c_{2} x \leftrightarrow \psi(x)$, and similarly with dissolution. An arbitrary computation of a $\mathrm{P}$ system consists of an arbitrary number of applications of non-dissolving rules and one application of a dissolving rule. Hence, the resulting vector sums up from the "initial" vector, one arbitrary "dissolving" vector, and an arbitrary linear combination of "non-dissolving" vectors.

It is worth noting that, by a similar reasoning, for a $\mathrm{P}$ system with multiple membranes, if the chronological order of dissolving membranes is fixed, the result is still $\mathbb{Z}^{d} S L I N_{\mathbb{N}}^{U}$. Indeed, each combination of rules (one for each catalyst) yields one vector, so all such possible combinations of non-dissolving rules yield a finite set of vectors, and multiple non-dissolving steps yield a linear set generated by these vectors. Thus, over the whole computation the result sums up from the initial configuration, a finite number of dissolution vectors, and a finite number of linear sets corresponding to the membrane structures reached during that computation. Since the total number of chronological orders of dissolving membranes is bounded, the known result already follows:

$$
Z^{d} O_{\mathbb{Z}} P_{*}\left(p c a t_{*}, \delta\right) \subseteq \mathbb{Z}^{d} S L I N_{\mathbb{N}} .
$$

Even with three membranes, in case two of them are elementary, the power of such purely catalytic P systems over integers is still $\mathbb{Z}^{d} S L I N_{\mathbb{N}}^{U}$, but for a different reason: each elementary membrane contributes with its uniform semilinear set, and a sum of two uniform semilinear sets is still uniform semilinear.

Let us now examine a $\mathrm{P}$ system with three nested membranes - the minimal number to obtain a set which is not in $\mathbb{Z}^{d} S L I N_{\mathbb{N}}^{U}$. Let the vector obtained by joining the initial contents of all membranes be $\mathbf{a}$, the set of non-dissolving vectors of the elementary membrane be $A_{3}$, the set of dissolving vectors of the elementary membrane be $B_{3}$, the sets of non-dissolving and dissolving vectors in the middle membrane associated to catalyst $c_{2}$ are $A_{2}$ and $B_{2}$, and the similar sets associated to catalyst $c_{3}$ (which will arrive from the elementary membrane) are $A$ and $B$. Let us see what the resulting vector set is built from, besides $\mathbf{a}$.

A non-dissolving computation in three membranes adds at each step (an element of) $A_{3}$ to the elementary membrane and (an element of) $A_{2}$ to the middle membrane. Eventually all objects will arrive to the skin, so the three-membrane phase of the computation will contribute by (an arbitrary element of) $\left\langle A_{2}+A_{3}\right\rangle_{\mathbb{N}}$.

Then there are two possibilities. If membrane 2 is dissolved first, then the system continues computing by only applying the rules in membrane 3 , and eventually dissolving membrane 3 , yielding $B_{2}+\left\langle A_{3}\right\rangle_{\mathbb{N}}+B_{3}$. However, if membrane 3 is dissolved first, then both catalysts are active in membrane 2, eventually dissolving it, yielding $B_{3}+\left\langle A_{2}+A\right\rangle_{\mathbb{N}}+\left(B_{2}+A \cup A_{2}+B \cup A+B\right)$. The expression in parentheses corresponds to applying at least one dissolving rule. Therefore, the set of integer vectors generated by such a purely catalytic P system over integers with three nested membranes is 


$$
M=\mathbf{a}+B_{3}+\left\langle A_{2}+A_{3}\right\rangle_{\mathbb{N}}+\left(B_{2}+\left\langle A_{3}\right\rangle_{\mathbb{N}} \cup\left\langle A_{2}+A\right\rangle_{\mathbb{N}}+\left(B_{2}+A \cup A_{2}+B \cup B_{2}+B\right)\right),
$$

and the power of all three-membrane purely catalytic $\mathrm{P}$ systems over integers, noting that the power of the nested case subsumes the power of the case with two elementary membranes, is

$$
Z^{d} O_{\mathbb{Z}} P_{1}\left(\text { pcat }_{*}, \delta\right)=\left\{M \mid \mathbf{a} \in \mathbb{Z}^{d}, A_{2}, A_{3}, B_{2}, B_{3}, A, B \in F I N\left(\mathbb{Z}^{d}\right)\right\},
$$

where $M$ is the expression above. Unfortunately, it is not obvious what can be simplified in it, except $B_{3}$ can subsume a. So we try to analyze it in details, possibly going into particular cases.

All terms in the expression $M$ are bounded except three: $\left\langle A_{3}+A_{2}\right\rangle_{\mathbb{N}},\left\langle A_{3}\right\rangle_{\mathbb{N}}$ and $\left\langle A+A_{2}\right\rangle_{\mathbb{N}}$. These terms are not independent, even though $A_{2}, A_{3}$ and $A$ are three independent finite sets of vectors. It is, however, possible to separate them in a particular case when $\left|A_{3}\right|=1$, choosing $A_{2}=-A_{3}$ and $A=C-A_{2}$. Since $A_{3}$ is a singleton, the identity $A_{3}-A_{3}=\{\mathbf{0}\}$ holds, so the three unbounded terms become $\langle\{\mathbf{0}\}\rangle_{\mathbb{N}},\left\langle A_{3}\right\rangle_{\mathbb{N}}$ and $\langle C\rangle_{\mathbb{N}}$, so we are getting close to obtaining a union of two particular linear (or even uniform semilinear) sets with different base vectors.

Indeed, if we choose $\mathbf{a}=\mathbf{0}, B_{3}=\{\mathbf{0}\}, B_{2}=\{\mathbf{0}\}, B=\{\mathbf{0}\}$ and $A_{3}=\{\mathbf{e}\}$, expression $M$ simplifies to $\langle\{\mathbf{e}\}\rangle_{\mathbb{N}} \cup\langle C\rangle_{\mathbb{N}}+(C+\{\mathbf{e}\} \cup\{\mathbf{0}\})$, which can be rewritten as $\langle\{\mathbf{e}\}\rangle_{\mathbb{N}} \cup\langle C\rangle_{\mathbb{N}} \cup\{\mathbf{e}\}\langle C\rangle_{\mathbb{N}}$.

Alternatively, to avoid dealing with the union of three cases when membrane 2 is divided last, if we choose $B_{2}=A_{2}$ and $B=A$, then the last parenthesis in the general expression of set $M$ becomes simply $A_{2}+A=C$. Choosing $\mathbf{a}=\mathbf{0}$, $B_{3}=\{\mathbf{0}\}$, and $A_{3}=\{\mathbf{e}\}$, expression $M$ simplifies to $\langle\{\mathbf{e}\}\rangle_{\mathbb{N}}-\{\mathbf{e}\} \cup\langle C\rangle_{\mathbb{N}}+C$. Since $\mathbf{0} \in\langle\{\mathbf{e}\}\rangle_{\mathbb{N}}-\{\mathbf{e}\}$ and $\langle C\rangle_{\mathbb{N}}+C \cup\{\mathbf{0}\}=\langle C\rangle_{\mathbb{N}}$, in this case we can rewrite $M$ to

$$
-\{\mathbf{e}\} \cup\langle\{\mathbf{e}\}\rangle_{\mathbb{N}} \cup\langle C\rangle_{\mathbb{N}},
$$

which is a union of any two homogeneous linear sets, such that the first one has only one generator, united with the opposite vector of that generator. Hence,

$$
Z^{d} O_{\mathbb{Z}} P_{n}(c a t, \delta) \supsetneq Z^{d} S L I N_{\mathbb{N}}^{U}, n \geq 3 .
$$

What if $B=\emptyset$, i.e., catalyst $c_{3}$ has no associated dissolution rules in region 2 ? Then the general expression of set $M$ is immediately simplified to

$$
M=\mathbf{a}+B_{2}+B_{3}+\left\langle A_{2}+A_{3}\right\rangle_{\mathbb{N}}+\left(\left\langle A_{3}\right\rangle_{\mathbb{N}} \cup\left\langle A_{2}+A\right\rangle_{\mathbb{N}}+A\right),
$$

and in our case of $A_{3}=\{\mathbf{e}\}, A_{2}=-\{\mathbf{e}\}$ and $A=C+\{\mathbf{e}\}, M$ becomes

$$
\mathbf{a}+B_{2}+B_{3}+\left(\langle\{\mathbf{e}\}\rangle_{\mathbb{N}} \cup\langle C\rangle_{\mathbb{N}}+C+\{\mathbf{e}\}\right),
$$

and choosing $\mathbf{a}+B_{2}+B_{3}=\{-\mathbf{e}\}$, and noticing that $C 0$ times is covered by $\mathbf{e} 0$ times and $\langle C\rangle_{\mathbb{N}}+C \cup\{\mathbf{0}\}=\langle C\rangle_{\mathbb{N}}$, we simplify $M$ to $\{-\mathbf{e}\} \cup\langle\{\mathbf{e}\}\rangle_{\mathbb{N}} \cup\langle C\rangle_{\mathbb{N}}$, i.e., an "almost clean union" we already obtained before. Finally, we notice that we can equivalently write it as

$$
\langle\{\mathbf{e}\},-\mathbf{e}\rangle_{\mathbb{N}} \cup\langle C\rangle_{\mathbb{N}}
$$

Continuing the current approach with more membranes would only result in more cases. 


\subsection{Communication}

We would like to remark that adding target indications to the regular objects should not increase the power of purely catalytic P systems over integers. Indeed, looking at a purely catalytic $\mathrm{P}$ system over integers, it is easily decidable which membranes will eventually be dissolved. Hence, the only question is whether the contents of a region specified by target, after possible dissolutions, will be in the output. There is no need to examine the future of a moved regular object, since the resources in purely catalytic $\mathrm{P}$ systems over integers are unbounded, and we can view this copy of a moved object as staying in that region until the end of the computation.

However, if also the catalysts are allowed to have target indications associated, it does make a difference. We claim the following characterizations.

$$
\begin{aligned}
& Z^{d} O_{\mathbb{Z}} P_{*}\left(\text { mpcat }_{k}, \text { tar }_{n}\right)=\mathbb{Z}^{d} S L I N_{\mathbb{N}}, k \geq 1, \\
& Z^{d} O_{\mathbb{Z}} P_{*}\left(\text { mpcat }_{k}+\delta\right)=\mathbb{Z}^{d} S L I N_{\mathbb{N}}, k \geq 1, \\
& Z^{d} O_{\mathbb{Z}} P_{*}\left(\text { mpcat }_{*}, \delta\right)=\mathbb{Z}^{d} S L I N_{\mathbb{N}},
\end{aligned}
$$

The upper bound in either case is easy to see because the number of possible arrangements of catalysts across the given membrane structure (and any possible structures obtained from it by membrane dissolutions) is bounded. Hence, purely catalytic P systems over integers with mobile catalysts are still not more powerful than blind vector-addition systems with states, which characterize $\mathbb{Z}^{*} S L I N_{\mathbb{N}}$, see [2]. We now proceed to $\supseteq$ inclusions.

Consider an arbitrary semilinear set $\bigcup_{1 \leq i \leq m}\left\langle A_{i}, b_{i}\right\rangle_{\mathbb{N}}$, where for each $i, 1 \leq i \leq$ $m, A_{i}$ is a finite set, $A_{i} \cup\left\{b_{i}\right\} \subseteq \mathbb{Z}^{d}$. We construct the following purely catalytic $\mathrm{P}$ system over integers

$$
\begin{aligned}
\Pi_{1} & =\left(O, C, \mu, w_{1}, \cdots, w_{2 m+1}, R_{1}, \cdots, R_{2 m+1}, i_{0}=1\right) \text { where } \\
O & =\left\{a_{i} \mid 1 \leq i \leq d\right\}, C=\{c\}, \\
\mu & =\left[\left[[]_{m+2}\right]_{2} \cdots\left[[]_{2 m+1}\right]_{m+1}\right]_{1}, \\
w_{1} & =c, w_{i+1}=\lambda, 1 \geq i \geq 2 m, \\
R_{1} & =\left\{c \rightarrow\left(c, i n_{i+1}\right) v_{i} \mid 1 \leq i \leq m, \psi\left(v_{i}\right)=b_{i}\right\}, \\
R_{i+1} & =\left\{c \rightarrow c(v, \text { out }) \mid \psi(v) \in A_{i}\right\} \cup\left\{c \rightarrow\left(c, i n_{m+i+1}\right)\right\}, 1 \leq i \leq m, \\
R_{m+i+1} & =\emptyset, 1 \leq i \leq m .
\end{aligned}
$$

The work of $\Pi_{1}$ consists of a non-deterministic choice of $i$-th linear set to generate, by moving catalyst $c$ into membrane $i+1$ and producing $b_{i}$. After sending to the skin an arbitrary combination of vectors from $A_{i}$, the catalyst enters membrane $m+i+1$ and the system halts.

The system $\Pi_{2}$ is obtained from $\Pi_{1}$ by replacing the sets $R_{i+1}$ of rules, $1 \leq$ $i \leq m$, by

$$
\left\{c \rightarrow c v \mid \psi(v) \in A_{i}\right\} \cup\left\{c \rightarrow\left(c, i n_{m+i+1}\right) \delta\right\} .
$$


It works just as $\Pi_{1}$, with one difference, Here, instead of sending $v$ out (possibly containing negative multiplicities), the linear combination of vectors from $A_{i}$ is generated directly in membrane $i+1$, and is released into the skin upon dissolution of membrane $i+1$, simultaneously with sending the catalyst into the elementary membrane $m+i+1$. Now consider the following purely catalytic $\mathrm{P}$ system over integers.

$$
\begin{aligned}
\Pi_{3} & =\left(O, C, \mu, w_{1}, \cdots, w_{3 m+1}, R_{1}, \cdots, R_{3 m+1}, i_{0}=1\right) \text { where } \\
O & =\left\{a_{i} \mid 1 \leq i \leq d\right\}, C=\left\{c_{i} \mid 1 \leq i \leq m+1\right\}, \\
\mu & =\left[\left[\left[[]_{2 m+2}\right]_{m+2}\right]_{2} \cdots\left[\left[[]_{3 m+1}\right]_{2 m+1}\right]_{m+1}\right]_{1}, \\
w_{1} & =c_{1}, w_{i+1}=\lambda, 1 \leq i \leq 2 m, \\
w_{2 m+1+i} & =c_{1+i}, 1 \geq i \geq m, \\
R_{1} & =\left\{c_{1} \rightarrow\left(c_{1}, i n_{i+1}\right) v_{i} \mid 1 \leq i \leq m, \psi\left(v_{i}\right)=b_{i}\right\}, \\
R_{i+1} & =\left\{c_{1} \rightarrow c_{1} v \mid \psi(v) \in A_{i}\right\} \\
& \cup\left\{c_{1} \rightarrow\left(c_{1}, i n_{m+i+1}\right), c_{i} \rightarrow c_{i} \delta\right\}, 1 \leq i \leq m, \\
R_{m+i+1} & =\left\{c_{1} \rightarrow\left(c_{1}, i n_{2 m+i+1}\right), c_{i} \rightarrow\left(c_{i}, \text { out }\right)\right\}, 1 \leq i \leq m, \\
R_{2 m+i+1} & =\left\{c_{1} \rightarrow c_{1} \delta\right\}, 1 \leq i \leq m .
\end{aligned}
$$

The basic idea is the same, but the implementation is a little longer. To each linear set $i, 1 \leq i \leq n$, three nested membranes are associated $(i+1, m+i+1$ and $2 m+i+1)$. The beginning is just like in the case of $\Pi_{2}$, until catalyst $c_{1}$ is sent into membrane $m+i+1$, but membrane $i+1$ is not dissolved yet. Then, $c_{1}$ enters the elementary membrane $2 m+i+1$ and dissolves it, releasing catalyst $c_{i+1}$ into the surrounding membrane $m+i+1$. Clearly, $c_{1}$ cannot reenter membrane $2 m+i+1$, which no longer exists, so it has no applicable associated rules. Catalyst $c_{i}$, however, is sent out to membrane $i+1$, and dissolves it, which releases all generated regular objects to the skin and halts the computation. This proves the characterizations.

\section{Conclusions}

We have reproved that the power of purely catalytic $\mathrm{P}$ systems over integers is contained in the family of all semilinear sets of vectors of integers. We then have shown that with one membrane purely catalytic $\mathrm{P}$ systems over integers give degenerate results, and with two membranes they are characterized exactly by the family of all uniform semilinear sets of vectors of integers. With more membranes, this equality becomes a strict inclusion, and a specific union of linear sets with different base vectors have been obtained. More specifically, for any vector $\mathbf{e} \in \mathbb{Z}^{d}$ and any finite set $C \subseteq \mathbb{Z}^{d}$, purely catalytic $\mathrm{P}$ systems over integers can generate

$$
\langle\{\mathbf{e}\},-\mathbf{e}\rangle \cup\langle C\rangle_{\mathbb{N}} .
$$


The most interesting open question remaining is whether $Z^{*} O_{\mathbb{Z}} P_{*}\left(p c a t_{*}, \delta\right)$ is closed under union. While in almost all cases in membrane computing closure under union is trivial, e.g., by making a non-deterministic choice in the first step of the computation, the current situation is rather surprising.

Finally, we have considered the variants with mobile catalysts, and showed a few combinations of features leading to characterizations of semilinear sets of $\mathbb{Z}$-vectors.

\section{References}

1. A. Alhazov, B. Aman, R. Freund, and Gh. Păun. Matter and anti-matter in membrane systems. In H. Jürgensen, J. Karhumäki, and A. Okhotin, editors, Descriptional Complexity of Formal Systems: 16th International Workshop, DCFS 2014, Turku, Finland, August 5-8, 2014. Proceedings, pages 65-76. Springer, 2014.

2. A. Alhazov, O. Belingheri, R. Freund, S. Ivanov, A. E. Porreca, and C. Zandron. Semilinear sets, register machines, and integer vector addition $(\mathrm{P})$ systems. This volume, 2016.

3. O. Belingheri, A. E. Porreca, and C. Zandron. P systems with hybrid sets, 2016. Workshop on Membrane Computing, submitted.

4. J. Carette, A. P. Sexton, V. Sorge, and S. M. Watt. Symbolic domain decomposition. In S. Autexier, J. Calmet, D. Delahaye, P. D. F. Ion, L. Rideau, R. Rioboo, and A. P. Sexton, editors, Intelligent Computer Mathematics, 10th International Conference, AISC 2010, 17th Symposium, Calculemus 2010, and 9th International Conference, MKM 2010, Paris, France, July 5-10, 2010. Proceedings, volume 6167 of Lecture Notes in Computer Science, pages 172-188. Springer, 2010.

5. R. Freund, O. Ibarra, Gh. Păun, and H.-C. Yen. Matrix languages, register machines, vector addition systems. Third Brainstorming Week on Membrane Computing, pages 155-167, 2005.

6. R. Freund, S. Ivanov, and S. Verlan. P systems with generalized multisets over totally ordered abelian groups. In Int. Conf. on Membrane Computing, volume 9504 of Lecture Notes in Computer Science, pages 117-136. Springer, 2015.

7. S. A. Greibach. Remarks on blind and partially blind one-way multicounter machines. Theoretical Computer Science, 7(3):311-324, 1978.

8. C. Haase and S. Halfon. Integer vector addition systems with states. In J. Ouaknine, I. Potapov, and J. Worrell, editors, Reachability Problems: 8th International Workshop, RP 2014, Oxford, UK, September 22-24, 2014. Proceedings, pages 112-124. Springer, 2014.

9. J. Hopcroft and J.-J. Pansiot. On the reachability problem for 5-dimensional vector addition systems. Theoretical Computer Science, 8(2):135-159, 1979.

10. S. N. Krishna and A. Păun. Results on catalytic and evolution-communication P systems. New Generation Computing, 22(4):377-394, 2004.

11. Gh. Păun. Some quick research topics. http://www.gcn.us.es/files/OpenProblems_bwmc15.pdf.

12. Gh. Păun. Computing with membranes. Journal of Computer and System Sciences, 61:108-143, 1998.

13. Gh. Păun, G. Rozenberg, and A. Salomaa. The Oxford Handbook of Membrane Computing. Oxford University Press, Inc., New York, NY, USA, 2010. 
